\title{
Plantas Medicinais e Fitoterapia em Oriximiná - Pará, Brasil: Percepção e Intenção de Uso pelos Profissionais do Sistema Único de Saúde (SUS)
}

\author{
Stela Souza Santos ${ }^{\text {a }}$ Paulo H. O. Léda ${ }^{\text {; }}$ Danilo Ribeiro de Oliveira ${ }^{\text {c,* }}$ \\ ${ }^{a}$ Secretaria Municipal de Saúde, Prefeitura Municipal de Oriximiná, Oriximiná, PA, Brasil \\ ${ }^{\mathrm{b}}$ Farmanguinhos, Fundação Oswaldo Cruz, Rio de Janeiro, RJ, Brasil \\ ${ }^{\mathrm{c}}$ Faculdade de Farmácia, Universidade Federal do Rio de Janeiro, Rio de Janeiro, RJ, Brasil.
}

\section{Histórico do Artigo \\ Recebido em: \\ $12 / 10 / 2017$ \\ Aceito em: \\ $09 / 02 / 2018$}

\section{Palavras-chave}

Terapias

complementares;

Sistema Único de Saúde

(SUS); gestão em

saúde; programas

nacionais de saúde.

\begin{abstract}
RESUMO
A Política Nacional de Plantas Medicinais e Fitoterápicos tem como diretrizes ampliar as opções terapêuticas, o uso sustentável da biodiversidade brasileira, valorizar e preservar o conhecimento de povos tradicionais, fortalecer a agricultura familiar, gerar emprego e renda, inclusão social, dentre outras. O presente estudo avaliou desafios e perspectivas à inserção da fitoterapia no SUS em Oriximiná-Pará. Para tal, foram entrevistados 65 profissionais de saúde de nível superior, vinculados ao SUS no Município. A maioria deles era do sexo feminino, de religião católica, não naturais de Oriximiná, atuantes tanto no setor público quanto no privado. Os mesmos destacaram mais pontos negativos no SUS do que positivos, como problemas de gestão, acompanhados pelo aumento da demanda. Além disso, outras questões levantadas mereceram destaque, tais como: 1- viabilidade de utilizar fitoterápicos em substituição a determinados medicamentos; 2- prática da automedicação, principalmente, através de chás caseiros e/ou alimentos funcionais; 3 - falta de treinamento, capacitação ou especialização sobre plantas medicinais e fitoterápicos; e 4- interesse em receber a capacitação necessária para atuar na fitoterapia. Desse modo, as informações obtidas incentivam a implantação de ações voltadas à fitoterapia, visto que a maior solicitação dos profissionais prescritores foi à realização de treinamentos específicos acerca do tema. No entanto, os desafios são muitos, a começar por obter a efetiva participação da Secretaria Municipal de Saúde e o engajamento multidisciplinar dos profissionais da rede pública de saúde, bem como a aplicação de recursos necessários para a viabilização das ações, atrelados a uma boa gestão dos recursos humanos e financeiros.
\end{abstract}

Medicinal Plants and Phytotherapy in Oriximiná - Pará, Brazil: Perception and Intention of Use by Professionals of the Unified Health System

\section{ABSTRACT}

The National Policy on Medicinal Plants and Herbs has as guidelines the expansion of therapeutic options and improvements in health care, sustainable use of Brazilian biodiversity, enhancement and preservation of the traditional knowledge associated, strengthening of agriculture family, generation of employment and income, social inclusion, among others. Therefore, the present study seeks to evaluate challenges and perspectives to the insertion of phytotherapy in the Public Health System (SUS) in Oriximiná-Pará. Most of them were female, of Catholic religion, unnatural of Oriximiná, and worked in both the public and private sectors. They highlighted more negative points in SUS than positives, such as management problems, accompanied by increased demand. In addition, other issues raised were highlighted, such as: 1 - feasibility of using herbal medicines to replace certain drugs; 2 - practice of self-medication, mainly through homemade teas and / or functional foods; 3- lack of training, capacity or specialization on medicinal plants and herbal medicines; and 4- interest in receiving the necessary training to act in herbal medicine. In this way, the information obtained encourages the implementation of actions directed to phytotherapy, since the greatest request of prescribing professionals was to carry out specific training on the topic. However, the challenges are many, starting with the effective participation of the Municipal Health Department and the multidisciplinary engagement of professionals in the public health network, as well as the application of resources necessary for the viability of actions, linked to good management human and financial resources.

\footnotetext{
*Autor correspondente: danilopharma@gmail.com (D. R. Oliveira)
} 


\section{Introdução}

O sucesso terapêutico dos fármacos para combater ou controlar doenças levou a um modelo de desenvolvimento apoiado na medicalização da sociedade, baseado no binômio normalidade-patologia (1), fundamento da medicina ocidental biomédica. $\mathrm{O}$ que, por sua vez, levou a um aumento da rigidez do sistema regulatório diante da necessidade de controlar a eficácia e a segurança dos fármacos sintéticos, formando a tríade da regulação sanitária, conhecida como segurança, eficácia e qualidade. Assim, a denominada "revolução química" acarretou na chamada "Reforma Científica e Terapêutica", liderada pelos EUA, no início do século XX, a qual estava baseada na ideia de que a ciência dos homens se poderia equiparar às criações da natureza por meio da síntese química, ou mesmo superá-las (2).

Nesse contexto, as plantas medicinais foram praticamente excluídas do sistema de saúde, sendo consideradas de pouca utilidade terapêutica, apesar da importância e da contribuição das mesmas para desenvolvimento e avanço da biomedicina (3). Isso também se refletiu na formação dos profissionais de saúde que também não tiveram acesso ao conhecimento acerca da fitoterapia, impossibilitando-os de receber uma visão crítica acerca do tema. Por outro lado, havia também a expectativa de que modelo biomédico fosse suficiente para o controle de todas as patologias (4). Entretanto, o crescimento populacional e das doenças associadas ao ambiente e/ou ao envelhecimento trouxeram a necessidade de compreender as possíveis interferências dessas relações na saúde individual ou coletiva que, por sua vez, são mais complexas do que o atual modelo apoiado exclusivamente no processo de medicalização da sociedade. Além disso, a maioria dos problemas de saúde apresentados pelas pessoas é simples, em que mais de $80 \%$ dos casos poderiam ser atendidos e resolvidos na atenção básica de saúde por meio do acolhimento e do estabelecimento de uma relação entre os agentes de saúde e a população atendida. Assim, compreender os processos de adoecimento vai muito além da simplificação habitual presentes nas dicotomias de causa e efeito (5).

De acordo com Luz (2007) (6), além de produzir medicamentos para controlar e erradicar patologias, há a necessidade de abordar questões socioculturais, as quais não são objeto de estudo de uma medicina altamente especializada e tecnificada. Por isso, houve a reinvindicação para que outras abordagens em saúde fossem reconhecidas nos sistemas oficiais de saúde (medicina tradicional chinesa, ayverdica, fitoterapia etc). Diante disso, a Organização Mundial da Saúde (OMS) recomenda que os países membros reconheçam e elaborem políticas para a introdução da medicina tradicional e seus recursos terapêuticos, especialmente as plantas medicinais e seus produtos, no âmbito sanitário e na atenção básica à saúde (7).

No Brasil, a criação do Sistema Único de Saúde (SUS) possibilitou a aprovação de regulamentos que incluem a participação da sociedade civil na construção de políticas de saúde, com ações para atenção e cuidado da saúde das famílias. Isso foi fundamental para a elaboração de políticas específicas para atender à incorporação da fitoterapia no SUS, a saber: a Política Nacional de Plantas Medicinais e Fitoterápicos (PNPMF) (8) e a Política Nacional de Práticas Integrativas e Complementares (PNPIC) (9). Nesse sentido, o MS adotou como modelo a ser implantado nos municípios a "Farmácia Viva", que respeita as características socioambientais e serviu como base para propor o uso das plantas regionais que podem ser utilizadas diretamente na atenção básica de saúde $(10,11)$. Como consequência, a Farmácia Viva possibilita a seleção das plantas já usadas pela população (regionalização), estimulando o diálogo entre os saberes populares e científicos (12).

Essa iniciativa das Farmácias Vivas, por sua vez, abriu espaço para a discussão e 
implantação de políticas de acesso aos fitoterápicos no SUS, aproveitando a imensa biodiversidade e a capacidade técnico-científica brasileira instalada na área. Contudo, torna-se necessário avaliar o interesse dos profissionais de saúde sobre o tema, considerando que a maioria deles não recebe nenhuma capacitação sobre plantas medicinais e fitoterápicos (PMF) durante a formação acadêmica $(13,14)$. Portanto, o presente estudo tem por objetivo identificar os desafios e as perspectivas para a inserção das PMFs no SUS de Oriximiná.

\section{Materiais e Métodos}

\subsection{Caracterização da área de estudo}

Oriximiná pertence à macrorregião do Baixo Amazonas Paraense, tendo como limites a Oeste o município de Faro e o Estado de Roraima, a Leste o município de Óbidos, ao Norte a Guiana e Suriname e, ao Sul, os Municípios de Juruti e Terra Santa. Possui cinco unidades de conservação (UCs): a Reserva Biológica (Rebio) do Rio Trombetas, a Floresta Nacional (Flona) Saracá-Taquera, as Florestas Estaduais (Flotas) Trombetas e Faro e a Estação Ecológica (ESEC) de Grão-Pará. Em 2015, a população estimada foi de 69.024 habitantes (15), a qual é constituída por povos indígenas, distribuídos em 16 aldeias e 151 comunidades (quilombolas, ribeirinhas e rurais). Quanto à rede de saúde, Oriximiná mantém 06 Unidades Básicas de Saúde (UBS) e 10 Postos de Saúde (PS) na área rural, três hospitais (dois públicos e um privado, dois Laboratórios de Análises Clínicas e uma Maternidade (16). As UBS atendem aos 17 bairros da área urbana, enquanto os PS atuam nas 171 comunidades distibuídas em seis grandes macroregiões, a saber: Alto Trombetas, Médio Trombetas, Baixo Trombetas, Erepecurú-cuminã, Sapucuá e Planalto $(16,17)$.

\subsection{Coleta de Dados}

Foi realizada uma pesquisa quali-quantitativa, de característica exploratória e descritiva, por meio de entrevistas, com aplicação de um formulário semiestruturado, buscando selecionar todos os profissionais de saúde de nível superior vinculados à Rede Pública de Saúde de Oriximiná. Foram levantados um total de 69 profissionais, distribuídos em 32 enfermeiros, 19 médicos, 09 odontólogos, 03 nutricionistas, 03 fisioterapeutas e 03 farmacêuticos.

O formulário consistia em 2 partes: o Perfil dos Profissionais de Saúde (idade, gênero, religião, formação superior, tempo de formação, pós-graduação, tempo de exercício da profissão no Município, locais de trabalho, vantagens e desvantagens de se trabalhar no SUS); e Percepção dos Profissionais de Saúde sobre as Plantas Medicinais e os Fitoterápicos no SUS (experiência, interesse, capacitação, prescrição, automedicação em Plantas Medicinais e Fitoterápicos, características regionais etc.).

As entrevistas foram realizadas entre novembro de 2012 e fevereiro de 2013, após a obtenção da autorização do Comitê de Ética em Pesquisa do Hospital Universitário Clementino Fraga Filho, da Universidade Federal do Rio de Janeiro (CEP/HUCFF/FM/UFRJ), sob Parecer $\mathrm{n}^{\mathrm{o}}$ 124.709, de 18.10.2012. A todos os entrevistados foi apresentado o Termo de Consentimento Livre e Esclarecido (TCLE), respeitando o direito de recusa e de interrupção da entrevista. 


\section{Resultados e Discussões}

\subsection{Perfil dos Profissionais de Saúde}

Dentre os 69 profissionais de saúde de nível superior identificados, não foram entrevistados os farmacêuticos, já que uma delas foi a que realizou a pesquisa de campo, não podendo ser concomitantemente pesquisadora e sujeito da pesquisa. Quanto aos demais, por atuarem exclusivamente na área das análises clínicas, sem vínculo direto com as ações da atenção básica e com os usuários do SUS, também não foram incluídos. Além deles, um médico não foi entrevistado por estar ausente do Município no período do estudo, totalizando-se, assim, 65 entrevistados (33 enfermeiros, 18 médicos, 09 odontólogos, 03 nutricionistas e 03 fisioterapeutas).

$\mathrm{Na}$ primeira parte do questionário, buscou-se avaliar alguns dados sobre o perfil dos profissionais de saúde, onde se verificou, inicialmente, o tempo de exercício da profissão e a qualificação profissional.

Os médicos eram os que possuíam maior tempo de atuação profissional (média de 14,6 anos), seguido pelos odontólogos (13,4 anos), enfermeiros (8,9 anos), nutricionistas (7,1 anos) e fisioterapeutas (5,3 anos).

$\mathrm{Na}$ qualificação profissional, destacaram-se os nutricionistas, visto que todos possuíam especialização (Nutrição Clínica e Saúde Coletiva), seguido pelos enfermeiros $(81,8 \%)$ (Administração Hospitalar, Saúde da Família, Epidemiologia, Saúde pública, Obstetrícia, Gestão e Auditoria e Emergência). Dentre os médicos, 50\% possuíam residência em Pediatria, Neurologia, Endocrinologia, Ginecologia e Obstetrícia, Otorrinolaringologia, Ortopedia e Cirurgia geral, enquanto que 16,7\% possuíam especialização em Saúde do Trabalhador e Saúde da Família. Para os fisioterapeutas, 66,7\% possuíam especialização em Fisiologia do Exercício e Trauma-ortopedia, enquanto 44,4\% dos odontólogos eram especializados em Prótese Dentária, Implantes e Gestão em Saúde.

$\mathrm{Na}$ questão de gênero, há predominância do sexo feminino dentre esses profissionais $(61,5 \%)$, influenciado, principalmente, pelas enfermeiras $(81,8 \%)$ e nutricionistas (100\%). Esses percentuais são próximos aos dados apresentados por Bruschini (2007) (18) que destacou que a enfermagem e a nutrição estavam dentre os "tradicionais guetos profissionais femininos".

Uma inversão desse panorama pode ser observada na questão do gênero para os demais profissionais de saúde, ocorrendo uma prevalência maior dos homens como médicos $(72 \%)$, odontólogos $(66,7 \%)$ e fisioterapeutas $(66,7 \%)$, embora o número de mulheres nessas profissões tenha crescido vertiginosamente nas últimas décadas no país, especialmente na medicina. Segundo dados do Conselho Federal de Medicina (19), há uma notória feminilização da medicina no Brasil, embora o mercado profissional ainda seja predominantemente masculino, devendo, assim, permanecer, por pelo menos, mais duas décadas.

A origem dos profissionais de saúde também pode ajudar a compreender melhor esse quadro de gênero, visto que $72,3 \%$ desses profissionais não nasceram ou cresceram em Oriximiná. Historicamente, a fixação no interior é considerada mais favorável aos homens, uma vez que muitos conseguem trazer ou constituir a família no interior e, assim, melhor se adaptar à nova realidade, enquanto as mulheres costumam enfrentar mais adversidades e preconceitos (20). Tal fato pode explicar a razão de a maior parte dos médicos ser de fora do município $(96,9 \%)$ e do sexo masculino (72\%). Essa discrepância também pode demonstrar a dificuldade de acesso à população do interior da Amazônia à educação formal para ingressar nos cursos de medicina. 
Quanto à religião, a maioria declarou ser católica (86\%), apresentando baixos percentuais para espiritas (6\%), evangélicos (5\%) e ateus (3\%). Esse dado chamou à atenção já que não reflete o crescimento do número de evangélicos em todo o Brasil, que vem sendo acompanhado de um decréscimo do número de católicos, em que a maior redução de praticantes do catolicismo registrada foi justamente na região Norte, que passaram de 71,3\% da população, em 2000, para 60,6\% em 2010 (15). Contudo, isso pode ser entendido pelo fato de Oriximiná ter forte tradições católicas na formação da sociedade atual desde a colonização portuguesa. Além disso, há uma particularidade em relação ao que é considerado "católico" na Amazônia. Segundo Soares (2013) (21), "é raro ouvir de alguém dessa região, com exceção dos próprios curadores, que sua religião seja a do candomblé, da umbanda ou da chamada "pajelança" amazônica, pois essas religiões, no contexto local, estão imersas numa visão de mundo que engloba o catolicismo professado".

Outra questão analisada foi sobre o setor de atuação desses profissionais, levando em consideração se os mesmos trabalhavam exclusivamente no setor público de saúde ou se conciliavam suas atividades com o setor privado. Foi verificado que apenas os enfermeiros entrevistados (100\%) trabalhavam exclusivamente no SUS, enquanto que os demais profissionais atuavam em ambos os setores: nutricionistas (50\%), médicos $(55,5 \%)$, odontólogos $(88,9 \%)$ e fisioterapeutas $(100 \%)$. Nesse contexto, é interessante ressaltar a sobrecarga de trabalho e a acumulação de empregos por parte dos profissionais de saúde no SUS. Esse quadro pode representar um fator limitante para a implementação da fitoterapia, visto que pode ser considerada mais uma atividade complementar e extra às suas atribuições, caracterizando um excesso de trabalho para muitos.

É interessante mencionar, ainda, o baixo número de profissionais de saúde disponíveis na rede pública de saúde para atender à população de Oriximiná. No caso dos médicos, por exemplo, a média local é muito inferior à média nacional de 2 médicos para cada mil habitantes, o que é comum em estados como Pará, Maranhão e Amapá (22).

Por fim, buscou-se avaliar qualitativamente as opiniões desses profissionais sobre as vantagens e desvantagens de se atuar no SUS de Oriximiná, merecendo destaque alguns pontos positivos:

1. Estabilidade no cargo, mencionada por todas as categorias;

2. Salário considerado bom ou melhor que o do setor privado, especialmente no caso dos médicos e enfermeiros;

3. O lado humanitário, destacado dentre os médicos e enfermeiros que relataram a importância do contato com os comunitários, a necessidade de contribuir para a sociedade, de trabalhar melhor a questão social, de atender todo o público, lidando com indivíduos de todas as rendas e das minorias;

4. O aperfeiçoamento profissional também foi considerado um ponto positivo para os médicos, enfermeiros e odontólogos;

5. Outras vantagens: Os enfermeiros citaram que a categoria tem mais autonomia no SUS, com facilidade de acesso aos Programas, além de monitoramento constante da saúde dos pacientes, enquanto os médicos destacaram o acesso gratuito à medicação, melhor acompanhamento no diagnóstico, possibilidade de atendimento familiar, uma boa rede de referência e contrarreferência e a diversidade de patologias encontradas. Para os fisioterapeutas, foi destacado a diversidade de patologias assistidas e a maior interação com os demais profissionais de saúde.

As desvantagens destacadas em atuar no SUS foram:

1. Demanda excessiva para o número de profissionais, também relacionada ao déficit de recursos humanos;

2. Falta de materiais, de equipamentos, insumos e medicamentos;

3. Problemas de gestão, tais como: falta de organização, demora no encaminhamento do 
paciente, falta de gerência financeira e de recursos, falta de protocolos clínicos e rotinas, demora na resolução dos problemas, morosidade/lentidão, desorganização, excesso de burocracia e demora no atendimento de pacientes transferidos para serviço de alta complexidade;

4. Desvalorização e falta de reconhecimento profissional, bem como a ausência de uma lotação fixa também foram relatadas pelos enfermeiros;

5. Inexistência de um plano de cargos e salários foi relatada por enfermeiros e odontólogos;

6. Falta de manutenção dos equipamentos é um problema destacado pelos odontólogos;

7. Perda de contato com o paciente, prejudicando a continuidade no atendimento, em alguns casos;

8. Indisciplina existente por parte de profissionais do SUS, bem como a falta de punições para más condutas foram destacadas por médicos;

9. "Fator amazônico" foi também considerado pelos médicos, uma vez que traz inúmeras dificuldades logísticas inerentes às distâncias, falta de transporte, gastos excessivos, longos períodos de deslocamento, dificultando o acesso tanto dos profissionais de saúde à população residente nas áreas rurais quanto o inverso.

Uma melhor compreensão do "fator amazônico" pode ser extraída do trabalho de Confalonieri (2005) (23), que destaca peculiaridades da paisagem e da geografia amazônica, tais como: "grande extensão territorial, vias de acesso predominantemente fluviais e a baixa densidade demográfica".

Embora seja interessante notar que os profissionais veem muitos pontos positivos em trabalhar no SUS em Oriximiná, por sua vez, é possível enumerar um número maior de problemas relatados, o que gera um alerta quanto à qualidade do serviço no SUS, especialmente, em função dos problemas de gestão que são acompanhados pelo aumento da demanda.

\subsection{Percepção dos profissionais de saúde sobre Plantas Medicinais e Fitoterápicos no SUS}

Na segunda parte do questionário, buscou-se verificar qual a percepção, conhecimento e interesse dos profissionais de saúde de nível superior sobre as plantas medicinais e os fitoterápicos (PMF). Inicialmente, foi feito um levantamento sobre a experiência e capacitação prévia desses profissionais, sendo verificado que poucos profissionais de medicina, enfermagem e odontologia haviam recebido algum tipo de treinamento sobre PMF, enquanto que nenhum nutricionista e fisioterapêuta tinham conhecimento sobre o tema. Todavia, a maioria dos entrevistados demonstrou interesse em receber qualificação em PMF e interesse em prescrevê-los, a exceção de alguns médicos $(22,2 \%)$ que se demonstram relutantes quanto à inserção deles próprios nessa prática terapêutica (Tabela 1).

Tabela 1: Perguntas relacionadas ao interesse, qualificação e prescrição de PMF por profissionais de saúde do SUS em Oriximiná.

\begin{tabular}{lccccc}
\hline \multirow{2}{*}{ Perguntas } & \multicolumn{5}{c}{ Categoria Profissional (\%) } \\
\cline { 2 - 7 } & Enf & Med & Odon & Nut & Fis \\
\hline Já recebeu treinamento em PMF? & 12,1 & 11,1 & 11,1 & 0 & 0 \\
\hline Tem interesse em receber treinamento sobre PMF? & 97 & 88,9 & 88,9 & 100 & 100 \\
\hline Tem interesse em prescrever PMF? & 97 & 77,8 & 100 & 100 & 100 \\
\hline Já fez automedicação com PMF? & 60,6 & 45,5 & 45,4 & 66,6 & 66,6 \\
\hline Já prescreveu PMF para pacientes? & 43,5 & 51,8 & 0 & 66,6 & 66,6 \\
\hline
\end{tabular}

$\mathrm{PMF}=$ plantas medicinais e fitoterápicos; Enf = enfermeiro(a); Med = médico(a); Odon = Odontólogo(a); Nut $=$ Nutricionista $;$ Fis $=$ fisioterapeuta

Esses resultados demonstraram-se promissores e apontaram a necessidade em se priorizar a qualificação dos profissionais de saúde do SUS para a inserção da fitoterapia 
como uma prática terapêutica complementar. Outra estratégia que tem sido aventada nos últimos anos, em esfera nacional, é a necessidade da inclusão de disciplinas específicas, como a 'Fitoterapia', dentre outras, nos currículos dos cursos de graduação da área de saúde. Assim, esses estudantes de saúde, quando formados, poderiam ter um mínimo de capacitação e interesse em atuar nessa prática terapêutica no SUS $(24,25)$.

Além disso, segundo as diretrizes da Política Nacional de Educação Permanente em Saúde (PNEPS) (26), deve-se partir da realidade dos serviços, vivenciada pelos trabalhadores, gestores e usuários, para se transformar o SUS, cuja integração entre o ensino/educação e o serviço/trabalho deve promover mudanças tanto no processo de formação quanto nas práticas de saúde. Para tanto, há necessidade de atender à diretriz do Programa Nacional de Plantas Medicinais e Fitoterápicos (PNPMF) (27) de "Promover capacitação técnica e educação permanente em plantas medicinais e fitoterápicos, dos profissionais de saúde do SUS, vigilantes e agentes comunitários, em conformidade com a PNEPS".

$\mathrm{Na}$ busca, ainda, de compreender o interesse e a vivência desses profissionais quanto às $\mathrm{PMF}$, foi questionado se eles faziam uso, independente de prescrição médica, ou se prescreviam esse recurso terapêutico, mesmo que de forma informal, por exemplo, para parentes e amigos, conforme descrito nas duas últimas questões da Tabela 1.

No quesito automedicação, quem mais se destacou foram nutricionistas, fisioterapeutas e enfermeiros. Contudo, os nutricionistas alegaram apenas uso de alimentos funcionais (por ex. couve para gastrite), enquanto os demais profissionais destacaram o uso de diversas PMFs (Quadro 1):

Quadro 1: Plantas Medicinais mencionadas por profissionais de nível superior do SUS no Município de Oriximiná-PA.

\begin{tabular}{|c|c|}
\hline Nome Popular & Nome Científico* \\
\hline abacate & Persea americana Mill. (Lauraceae) \\
\hline alfavaca & Ocimum campechianum Mill. (Lamiaceae) \\
\hline algodão-roxo & Gossypium barbadense L. (Malvaceae) \\
\hline alho (cápsulas) ${ }^{* *}$ & Allium sativum L. (Liliaceae) \\
\hline andiroba & Carapa guianensis Aubl. (Meliaceae) \\
\hline barbatimão & Stryphnodendron adstringens (Mart.) Coville (Fabaceae) \\
\hline berinjela & Solanum melongena $\mathrm{L}$. \\
\hline boldo & Plectranthus barbatus (Andrews) Benth. (Lamiaceae) \\
\hline canela $^{* *}$ & Cinnamomum verum J. S. Presl. (Lauraceae) \\
\hline catuaba & Não identificada \\
\hline camomila $^{* *}$ & Matricaria chamomilla L. (Asteraceae) \\
\hline capim-limão & Cymbopogon citratus (DC.) (Poaceae) \\
\hline cimicifuga $^{* *}$ & Actaea racemosa L. (Ranunculaceae) \\
\hline copaíba & Copaifera sp. (Fabaceae) \\
\hline erva-cidreira & Lippia alba (Mill.) N.E. Brown (Verbenaceae) \\
\hline erva-doce $^{* *}$ & Pimpinella anisum L. (Apiaceae) \\
\hline eucalipto $^{* *}$ & Eucalyptus globulus Labill. (Myrtaceae) \\
\hline folha-grossa & Bryophyllum calycinum Salisb. (Crassulaceae) \\
\hline genipapo & Genipa americana L. (Rubiaceae) \\
\hline ginko $^{* * *}$ & Ginkgo biloba L. (Ginkgoaceae) \\
\hline goiabeira (broto) & Psidium guajava (L.) Radd. (Myrtaceae) \\
\hline graviola & Annona muricata L. (Annonaceae) \\
\hline hortelãzinho & Mentha sp. (Lamiaceae) \\
\hline jucá & Caesalpinia ferrea Mart. (Fabaceae) \\
\hline Linhaça $^{* *}$ & Linum unisatissimum L. (Linaceae) \\
\hline mamão (semente) & Carica papaya L. (Caricaceae) \\
\hline maracujá peroba $^{* *}$ & Passiflora edulis Sims (Passifloraceae) \\
\hline pata-de-vaca & Bauhinia spp. (Fabaceae) \\
\hline peão-branco & Jatropha curcas L. (Euphorbiaceae) \\
\hline piquiá & Caryocar villosum (Aubl.) Pers. (Caryocaraceae) \\
\hline quebra-pedra & Phyllanthus spp. (Euphorbiaceae) \\
\hline valeriana $^{* *}$ & Valeriana officinalis L. (Caprifoliaceae) \\
\hline
\end{tabular}

* Os nomes científicos utilizados foram obtidos das referências: Oliveira, 2002 (28); Oliveira, 2009 (29); IN 02/2014 (30);

${ }^{* *}$ Espécies vegetais com segurança e efetividade baseados na literatura técnico-científica ou por estudos clínicos de segurança e eficácia, presentes no anexo da IN 02/2014 (30) ou dentre as monografias de fitoterápicos de uso bem estabelecido da Comunidade Europeia (Community herbal monographs with well-established use), da Agência Europeia de Medicamentos (EMA) (31). 
Com exceção dos odontólogos, todos os profissionais de saúde mencionaram já ter prescrito, indicado e usado PMF com os pacientes (Tabela 1). Os nutricionistas destacaram orientar o uso de alimentos funcionais, enquanto que os fisioterapeutas deram destaque ao uso tópico de óleos (copaíba, andiroba e piquiá) e banhas medicinais, que é uma prática extremamente comum para o tratamento tópico de dores e inflamações na região amazônica (33). Alguns enfermeiros ainda alegaram já ter recomendado o uso de: pata de vaca (diabetes); quebra-pedra (com talco para assaduras); alfavaca; hortelã (chá); copaíba e andiroba (anti-inflamatórias); folhagrossa, algodão-roxo, leite do peão-branco e mel (xarope para garganta).

Alguns profissionais destacaram, ainda, que pelo fato de serem nativos da região amazônica, já tinham tradição familiar do uso das plantas medicinais. Outros também lembraram que a interação dos profissionais de saúde com membros de comunidades tradicionais era constante, seja com a ida de equipes de saúde até as comunidades, ou pela vinda deles à cidade, o que também despertava curiosidade, interesse e proximidade ao tema das plantas medicinais.

Mais da metade dos médicos afirmou já ter prescrito fitoterápicos, merecendo destaque para passiflora e valeriana como ansiolíticos, Ginkgo biloba para memória e cimicífuga para transtornos da menopausa. Esse número é considerado relevante, pois o ato da prescrição pelo médico tem um caráter de suma importância para a credibilidade junto ao sistema de saúde.

Houve também o relato de alguns médicos e enfermeiros de que a prescrição de PMF é restrita apenas ao seu núcleo familiar:

$$
\begin{aligned}
& \text { "Prescrevo para meus filhos", } \\
& \text { "Prescrevo somente para minha familia”, } \\
& \text { "Os parentes em casa sim, sempre passo", }
\end{aligned}
$$

Esse resultado foi semelhante ao de Rosa et al. (2011) (24), em que 44,5\% dos prescritores médicos relataram utilizar PMF em suas vidas pessoais.

Cabe ressaltar que dentre as 32 plantas mencionadas espontaneamente pelos entrevistados, apenas 10 (31,2\%) possuem dados de segurança e efetividade mínimos, fazendo parte de listas positivas de espécies vegetais $(30,31)$, que podem ser notificadas ou registradas como fitoterápicos, de acordo com a RDC 26/2014 (32). Oliveira et al. (2016) (33), justifica esse fato, principalmente, em função da ausência de estudos de segurança e eficácia para a maioria das espécies nativas, fazendo com que a vasta biodiversidade e o conhecimento tradicional associado às plantas medicinais brasileiras fiquem subutilizados frente ao seu enorme potencial terapêutico.

Nesse contexto, é interessante observar que, embora a maioria dos profissionais tenha alegado falta de qualificação para não fazer automedicação ou prescrição de PMF, conforme descrito na Tabela 2, alguns médicos e enfermeiros destacaram o desinteresse e a falta de confiança na fitoterapia como prática terapêutica, conforme alguns relatos críticos descritos a seguir:

\footnotetext{
"Não tem comprovação científica";

"Há necessidade de mais pesquisas que legitimem os princípios ativos";

"Depende da crença de quem prescreve e usa";

"Eficácia pelos relatos, segurança não!’.
}

Além disso, há uma insegurança no ato da prescrição, acrescido do caráter jurídico, a Resolução 1.499 do Conselho Federal de Medicina, que estabelece o veto à utilização de terapias não comprovadas cientificamente (25). 
Tabela 2: Justificativas para não realizar o uso e a prescrição de PMF pelos profissionais de saúde do SUS de Oriximiná-PA.

\begin{tabular}{ccccccccc}
\hline \multirow{2}{*}{ Profissionais } & \multicolumn{3}{c}{$\begin{array}{c}\text { Por quê não fazem automedicação } \\
\text { com PMF? } \\
(\boldsymbol{\%})\end{array}$} & \multicolumn{4}{c}{$\begin{array}{c}\text { Por que não tem interesse em } \\
\text { Prescrever PMF? } \\
(\boldsymbol{\%})\end{array}$} \\
\cline { 2 - 10 } & FQ & FI & FC & O & FQ & FI & FC & O \\
\hline Enfermeiros & 21,2 & 15,1 & 6,1 & 3 & 33,3 & 3 & 6,1 & 3 \\
\hline Médicos & 38,9 & 11,1 & 16,7 & 0 & 50 & 11,1 & 16,7 & 0 \\
\hline Odontólogos & 55,6 & 0 & 0 & 0 & 88,9 & 1,1 & 0 & 0 \\
\hline Nutricionista & 100 & 0 & 0 & 0 & 100 & 0 & 0 & 0 \\
\hline Fisioterapeuta & 100 & 0 & 0 & 0 & 66,6 & 0 & 0 & 33,3 \\
\hline
\end{tabular}

FQ = Falta Qualificação; FI = Falta de Interesse; FC = Falta de Confiança; $\mathrm{O}=$ Outros.

Buscou-se, ainda, levantar outras informações relacionadas com o uso e com as dúvidas da população, que pudessem ser trocadas entre os usuários do SUS de Oriximiná e os profissionais de saúde sobre as PMF. É interessante notar que a maioria dos profissionais de saúde entrevistados informou já ter ouvido dos pacientes relatos de cura pelo uso de plantas medicinais (Tabela 3). Muitos ainda disseram quais doenças eram informadas pelos pacientes: infecções de garganta (óleo de copaíba), diabetes (pata de vaca), pedra nos rins (quabra-pedra), ferida (barbatimão), picada de cobra (barbatimão), câncer (graviola), gastrites, hipertensão, tosse, hipercolesterolemia, hipertrigliceremia, dentre outras.

Tabela 3: Perguntas para os profissionais de Saúde de Oriximiná relacionadas aos pacientes do SUS.

\begin{tabular}{|c|c|c|c|c|c|c|}
\hline \multirow{2}{*}{\multicolumn{2}{|c|}{ Perguntas }} & \multicolumn{5}{|c|}{ Categoria Profissional (\%) } \\
\hline & & Enf & Med & Odon & Nut & Fis \\
\hline \multicolumn{2}{|c|}{$\begin{array}{l}\text { Já ouviu relatos de cura com PMF de } \\
\text { pacientes? }\end{array}$} & 75,9 & 72,2 & 100 & 66,7 & 100 \\
\hline \multicolumn{2}{|c|}{$\begin{array}{l}\text { Já ouviu relatos de reações adversas de PMF } \\
\text { por pacientes? }\end{array}$} & 26,1 & 50 & 22,2 & 0 & 0 \\
\hline \multicolumn{2}{|c|}{$\begin{array}{l}\text { Já ouviu dúvidas de pacientes sobre uso de } \\
\text { PMF? }\end{array}$} & 60,6 & 61,1 & 22,2 & 66.7 & 100 \\
\hline \multirow{3}{*}{$\begin{array}{l}\text { Tipos } \\
\text { de } \\
\text { dúvidas? }\end{array}$} & Sobre associação com medicamentos & 18,2 & 55,6 & 14,3 & 66,7 & 66,7 \\
\hline & Riscos à saúde/fazer mal & 30,3 & 22,2 & 14,3 & 33,7 & 33,7 \\
\hline & Outras & 13,1 & 0 & 0 & 0 & 0 \\
\hline \multicolumn{2}{|c|}{$\begin{array}{l}\text { Já ouviu relatos de reações adversas de PMF } \\
\text { por pacientes? }\end{array}$} & 26,1 & 50 & 22,2 & 0 & 0 \\
\hline
\end{tabular}

Enf = enfermeiro(a); Med = médico(a); Odon = Odontólogo(a); Nut = Nutricionista; Fis = fisioterapeuta

Da amostra obtida na pesquisa, a maior parte dos profissionais de saúde, com exceção dos dentistas $(22,2 \%)$, afirmou já ter ouvido dos usuários relatos de dúvidas acerca do uso de plantas medicinais, as quais estavam relacionadas, sobretudo, à associação de plantas com medicamentos e aos riscos à saúde (Tabela 3).

Reações adversas com PMF pelos pacientes foram mencionadas apenas por um pequeno número de enfermeiros e odontólogos, e por metade dos médicos entrevistados (Tabela 3), sendo relacionadas, principalmente, com plantas abortivas, tais como hemorragia uterina. Outras reações adversas relatadas foram: urticária, vômito, sonolência, pressão alta, dor de cabeça e sangramento nasal. É interessante notar que, na região amazônica, é comum e frequente o uso de uma planta tóxica, conhecida como cabaçinha ou buchinha do norte (Luffa operculata), para promover o aborto e para tratar sinusite, sendo possível que parte dos relatos de hemorragia uterina e de sangramento nasal sejam relacionados a ela $(29,35)$. O desconhecimento do efeito tóxico e de 
reações adversas na automedicação com plantas medicinais é uma realidade para a maioria da população brasileira. Esse fato poderia ser minimizado se os profissionais de saúde fossem qualificados para tal e se a população relatasse o uso das plantas e os seus efeitos colaterais. Uma importante contribuição ao registro das reações adversas pode-se dar através do Sistema de Notificações em Vigilância Sanitária (36). Contudo, é importante que haja uma conscientização dos profissionais de saúde para ocorrer a notificação de modo a contribuir para a saúde da população. Por outro lado, muitos pacientes não relatam que usam PMF concominantemente com outros medicamentos, inviabilizando a melhor orientação aos pacientes (37).

Por fim, na última parte da entrevista, buscou-se fazer perguntas diretamente relacionadas à inserção da fitoterapia em Oriximiná. Considerando que essa inserção faz parte das diretrizes da PNPMF, inicialmente, foi verificado o conhecimento dos profissionais de saúde sobre essa Política. Constatou-se que apenas um pequeno número de enfermeiros, médicos e dentistas já tinha algum conhecimento sobre ela (Tabela 4), o que termina sendo um fato negativo em virtude de que a PNPMF é utilizada como base para ações de capacitação e implantação da fitoterapia no SUS junto aos gestores municipais ou estaduais de saúde. Por sua vez, pesquisas demostram que profissionais prescritores, em particular os médicos, têm pouco conhecimento sobre as terapias complementares, mesmo tendo interesse em praticá-las $(13,14,25)$.

Quanto à oferta de PMF no SUS, a maioria dos profissionais de saúde considera uma boa alternativa, especialmente pela diversificação das opções terapêuticas. Contudo, um pequeno percentual de enfermeiros, médicos e odontólogos a consideraram desnecessária ou irrelevante (Tabela 4). Tal fato poderia ser explicado pelo receio do aumento de custos e problemas/dificuldades da gestão financeira dos recursos públicos destinados ao SUS local, ou até mesmo pelo receio de aumento de demanda de trabalho e atividades.

Tabela 4: Perguntas para os profissionais de Saúde sobre a inserção da Fitoterapia no SUS de Oriximiná.

\begin{tabular}{|c|c|c|c|c|c|c|}
\hline \multirow{2}{*}{\multicolumn{2}{|c|}{ Perguntas }} & \multicolumn{5}{|c|}{ Categoria Profissional (\%) } \\
\hline & & Enf & Med & Odon & Nut & Fis \\
\hline \multicolumn{2}{|c|}{ Conhece ou já ouviu falar da PMPMF? } & 30,4 & 33,3 & 11,1 & - & - \\
\hline \multicolumn{2}{|c|}{ É a favor da oferta de PMF no SUS? } & 97 & 83,3 & 88,9 & 100 & 100 \\
\hline \multicolumn{2}{|c|}{$\begin{array}{l}\text { PMF podem substituir determinados medicamentos } \\
\text { em alguns casos no SUS? }\end{array}$} & 81,8 & 77,8 & 88,9 & 100 & 100 \\
\hline \multicolumn{2}{|c|}{$\begin{array}{l}\text { Oriximiná tem vocação para a inserção das PMF no } \\
\text { SUS? }\end{array}$} & 78,7 & 88,9 & 77,7 & 100 & 100 \\
\hline \multicolumn{2}{|c|}{$\begin{array}{l}\text { As informações seculares do uso medicinal das plantas } \\
\text { servem como parâmetro de eficácia e segurança? }\end{array}$} & 69,7 & 61,1 & 88,9 & 66,7 & 66,7 \\
\hline \multirow{4}{*}{$\begin{array}{l}\text { A biodiversidade } \\
\text { local e as } \\
\text { comunidades } \\
\text { tradicionais de } \\
\text { Oriximiná } \\
\text { influenciam na } \\
\text { inserção das } \\
\text { PMFs no SUS? }\end{array}$} & Importante/ fundamental & 69,7 & 61,1 & 88,9 & 66,7 & 100 \\
\hline & Diferencial Regional/Estratégico & 63,6 & 44,4 & 55,6 & - & - \\
\hline & Indiferente & 6,1 & 5,6 & - & 33,3 & - \\
\hline & Outros & 15,1 & 11,1 & - & - & - \\
\hline \multirow{4}{*}{$\begin{array}{l}\text { O que é essencial } \\
\text { para a inserção } \\
\text { da Fitoterapia no } \\
\text { SUS em } \\
\text { Oriximiná? }\end{array}$} & Interdisciplinaridade & 33,3 & 38,9 & 55,5 & 33,3 & 100 \\
\hline & $\begin{array}{l}\text { Engajamento da SMS e dos } \\
\text { Profissionais } \\
\end{array}$ & 69,7 & 77,8 & 77,8 & 100 & 100 \\
\hline & Recursos & 45,5 & 50 & 66,7 & - & 100 \\
\hline & Outros & 15,1 & 16,7 & 22.2 & - & 33,3 \\
\hline
\end{tabular}

$\mathrm{E}=$ enfermeiro(a); $\mathrm{M}=$ médico(a); $\mathrm{O}=$ Odontólogo(a); $\mathrm{N}=$ Nutricionista; $\mathrm{F}=$ fisioterapeuta; ${ }^{*}$ Para essas questões, os entrevistados tiveram mais de uma opção de resposta. 
Sobre utilizar PMF em substutuição a determinados medicamentos em alguns casos no SUS, as respostas foram bem favoráveis para a maioria dos profissionais de saúde (Tabela 4), merecendo destaque alegações de uso para tratamento de tosse e catarro, ansiedade, distúrbios vasculares, desordens estomacais, intestinais e hepáticas, cólicas, flatulências (gases) e inflamações externas. Alguns ainda relataram a possibilidade de PMF para tratamento de distúrbios do climatério, dores e inflamações em geral, gastrite, ameba, diabetes, hipercolesterolemia, hipertensão e infecções primárias leves.

Contudo, alguns profissionais veem a fitoterapia apenas como uma terapia complementar ou, até mesmo, com algum desprezo, conforme relatos transcritos a seguir:

\footnotetext{
"PMF pode somar ao tratamento e não substituí-lo";

"a maioria das doenças são autolimitadas, desaparecem espontaneamente. Então, pode usar como um placebo, pois, em 5-7 dias, o paciente naturalmente já ficará bom".
}

Por sua vez, a maioria dos profissionais de saúde consideram o município de Oriximiná propício e vocacionado para a inserção da fitoterapia no SUS (Tabela 4). A impressão dos mesmos pode ser bem ilustrada através dos seguintes relatos:

\footnotetext{
"Bem feito, bem orientado, colocando ciência nesta arte, vale a pena. Afinal, a população já faz isso de forma rudimentar";

"É seguir a crença do povo";

"É cultural, as pessoas têm muito conhecimento, principalmente os antigos. Tem que ser valorizado";

"Há diversidade de recursos naturais e os profissionais poderão usar de forma preventiva";

"Além do baixo do custo, a comunidade vai aceitar bem. Já é uma prática deles";

"Nossa região é distante dos grandes centros e a cultura é propicia para as PMF”;

"O povo é habituado a usar e vai aderir";

"Vai ter aceitabilidade da população. Já existe o uso como questão cultural. É característico da região”;

"Já têm muitas pesquisas nesta área das PMFs das universidades que também ajudam”;

"As condições socioeconômicas do município são propícias em termos de custo. É bem mais barato. Além disso, já são cultivadas em muitas residências";

"Estamos cercados de áreas verdes, cheio de plantas medicinais";

"Muitos médicos vão aderir e a maioria das enfermeiras também";

"Têm profissionais interessados e isso pode estimular os pacientes".
}

Sobre as informações seculares do uso de plantas nativas no tratamento de doenças, os profissionais demostraram reconhecer a importância das mesmas (Tabela 4). Esse fato pode ser explicitado nos seguintes discursos:

\footnotetext{
"Vários medicamentos surgiram daî";

"Existem muitos relatos de cura com plantas medicinais...";

"Um povo não sobreviveria milênios sem nada para curar";

"A medicina da China é de uma cultura milenar e, atualmente, a acupuntura é uma especialidade médica, por que não valorizar a nossa?”;

"Serve de base para o aprofundamento cientifico".
}

A importância da biodiversidade e das comunidades locais para a implantação da Fitoterapia no SUS foi ressaltada pela maioria dos profissionais de saúde. Muitos consideraram ainda um diferencial regional e um fator estratégico (Tabela 4). Nesse 
sentido, algumas considerações dos entrevistados a respeito são aqui transcritas:

\begin{abstract}
"O conhecimento popular é super importante. Eles podem usar o que for cultivado";

"A população tem o que plantar. É importante envolver as comunidades";

"O deslocamento para entrada de medicamentos em Oriximiná é dificil. Os fitoterápicos representariam o melhor acesso aos medicamentos para os usuários".
\end{abstract}

Contudo, há profissionais indiferentes a esses fatores, dentre eles um médico que alegou que "Eles já perderam a cultura. Já chegam até induzindo a prescrição de medicamentos industrializados".

Por fim, os entrevistados opinaram sobre os fatores essenciais para a implantação da fitoterapia (Tabela 4). Os principais fatores foram: engajamento da Secretaria Municipal de Saúde e dos Profissionais $(89,1 \%)$, aplicação de recursos $(61,8 \%)$ e a interdisciplinaridade $(49,1 \%)$. Contudo, alguns profissionais destacaram outros pontos extremamente necessários como: continuidade do serviço; treinamento e capacitação; repasse de informação científica; memento terapêutico; ações educativas para a população aderir; engajamento de todos os profissionais; boa gestão; e participação das comunidades junto às pesquisas e ações.

O questionário foi finalizado com uma pergunta aberta para os entrevistados sobre "Como você enxerga o Papel da Assistência Farmacêutica em Oriximiná para a inserção da Fitoterapia no SUS?". A maioria dos entrevistados destacou a importância do farmacêutico para a implantação da fitoterapia, conforme exemplificado a seguir:

\footnotetext{
"A Fitoterapia no SUS tem que caminhar em conjunto com a Assistência Farmacêutica para ter mais segurança";

"O farmacêutico é o profissional mais qualificado para planejar, organizar, avaliar e identificar as potencialidades e multiplicar conhecimentos $e$ práticas para a inserção de um programa local com PMF";

"Geralmente, são os primeiros a se envolverem e acreditar na PMF no SUS";

"O conhecimento farmacêutico, associado à medicina natural, poderia ajudar a reverter as faltas de medicamentos na rede";

"Apesar de ser um produto natural, continua sendo um fármaco. Não pode deixar de ter uma assistência";

"É o regulador, a referência no Município, o profissional que tem esta incubência e responsabilidade na própria formação";

"São os profissionais mais qualificados, importantes no SUS e no acompanhamento e gestão do programa";

"Os pacientes usam e seria importante controlar e melhorar a adesão terapêutica";

"Os farmacêuticos estudam mais isso do que os médicos, possuem disciplinas de farmacobotânica e outras relacionadas às plantas medicinais";

"É o pilar do programa, as orientações viriam da Assistência Farmacêutica".
}

\title{
4. Considerações Finais
}

O Ministério da Saúde tem avançado no sentido de fortalecer as práticas integrativas e complementares (PICs) no Brasil com a adoção de uma política específica, a PNPIC. Essa política, juntamento com a PNPMF, busca integrar a fitoterapia aos programas da atenção básica de saúde. Portanto, um diagnóstico do perfil dos prescritores do SUS, e do interesse dos mesmos na prescrição de plantas medicinais e fitoterápicos é extremamente relevante para a implantação de um programa municipal, sendo 
imprescindível analisar e compreender as limitações e vocações locais (38). Nesse contexto, os resultados obtidos nessa pesquisa fornecem subsídios para a implementação de ações voltadas para a fitoterapia em Oriximiná na medida em que demonstra o perfil de conhecimento e de interesse dos profissionais de saúde acerca do tema.

Desse modo, as informações obtidas trouxeram incentivo em relação à implantação de ações voltadas para a fitoterapia, visto que a maior solicitação dos profissionais prescritores foi a realização de treinamentos específicos acerca do tema. No entanto, os desafios são muitos, a começar por obter a efetiva participação da Secretaria Municipal de Saúde e o engajamento multidisciplinar dos profissionais da rede pública de saúde, bem como a aplicação de recursos necessários para a viabilização das ações, atrelados a uma boa gestão dos recursos humanos e financeiros.

\section{Agradecimentos}

À Secretaria Municipal de Saúde de Oriximiná, especialmente àqueles que aceitaram participar desta pesquisa.

\section{Referências}

1. Nascimento MC, Barros NF, Nogueira M I, Luz MT. A categoria racionalidade médica e uma nova epistemologia em saúde. Ciênc Saúde Coletiva 2013; 18(12):3595-3604.

2. Sá IM. A resistência à cloroquina e a busca de antimalariais entre as décadas de 1960 e 1980 . Hist Ciênc Saúde-Manguinhos 2011; 18(2):407-430.

3. Yunes RA, Calixto JB. Plantas medicinais sob a ótica da moderna química medicinal. Chapecó: Argos; 2001.

4. Luz MT. Cultura contemporânea e medicinas alternativas: novos paradigmas em saúde no fim do século XX. Physis: Rev. Saúde Coletiva 2005; 15(Supl.):145-176.

5. Paim J. O que é o SUS. Rio de Janeiro: Editora FIOCRUZ; 2009.

6. Luz MT. Novos saberes e práticas em saúde coletiva. Estudo sobre racionalidades médicas e atividades corporais. $3^{\text {a }}$ edição. São Paulo: Hucitec; 2007.

7. Organización Mundial de la Salud. Estrategia de la OMS sobre medicina tradicional 2002-2005. Geneva: Organización Mundial de la Salud; 2002.

8. Brasil. Ministério da Saúde. Decreto $n^{\circ} 5.813$, de 22 de junho de 2006. Aprova a Política Nacional de Plantas Medicinais e Fitoterápicos e dá outras providências. Diário Oficial da União 2006; 23 jun.

9. Brasil. Ministério da Saúde. Portaria 971, de 3 de maio de 2006. Aprova a Política Nacional de Práticas Integrativas e Complementares (PNPIC) no Sistema Único de Saúde. Diário Oficial da União 2006; 4 mai.

10. Brasil. Ministério da Saúde. Portaria GM/MS nº 886, de 20 de abril de 2010. Institui a Farmácia Viva no âmbito do Sistema Único de Saúde (SUS). Diário oficial da União 2010; 22 abr.

11. Brasil. Ministério da Saúde, Anvisa. RDC No 18, de 03 de abril de 2013. Dispõe sobre as boas práticas de processamento e armazenamento de plantas medicinais, preparação e dispensação de produtos magistrais e oficinais de plantas medicinais e fitoterápicos em farmácias vivas no âmbito do Sistema Único de Saúde (SUS). Diário Oficial da União 2013; 4 abr.

12. Araújo M. Das ervas medicinais à fitoterapia. São Paulo: Ateliê Editorial/Fapesp; 2002.

13. Thiago SC, Tesser CD. Percepção de médicos e enfermeiros na Estratégia de Saúde da Família sobre terapias complementares. Rev Saúde Pública 2011;45(2):249-57.

14. Bruning MCR, Mosegui GBG, Vianna CMM. A utilização da fitoterapia e de plantas medicinais em unidades básicas de saúde nos municípios de Cascavel e Foz do Iguaçu-Paraná: a visão dos profissionais de saúde. Cienc Saúde Coletiva 2012; 17:2675-2685.

15. Instituto Brasileiro de Geografia e Estatística - IBGE. Censo Demográfico 2016. [acessado 201618 ago]. Disponível em: http://www.cidades.ibge.gov.br 
16. Mineração Rio do Norte - MRN. Programa Territórios Sustentáveis. Gestão Integrada na Amazônia. Diagnóstico da Gestão Pública de Oriximiná - PA. Relatório 2015.

17. Prefeitura Municipal de Oriximiná/Secretaria Municipal de Saúde - PMO/SMS. Relatório de Gestão da Secretaria Municipal de Saúde de Oriximiná-Pará, Relatório 2011.

18. Bruschini MCA. Trabalho e gênero no Brasil nos últimos dez anos. Cad Pesqui 2007; 37(132): 537-572.

19. Conselho Federal de Medicina (CFM/CREMESP). Demografia Médica no Brasil. Vol. 1. Dados Gerais e Descrições de Desigualdades. Relatório de Pesquisa. 2011. [Acessado 2016 set 29] Disponível em: http://portal.cfm.org.br/images/stories/pdf/demografiamedicanobrasil.pdf

20. Justo WR, Neto RDMS. Quem são e para onde vão os migrantes no Brasil? O perfil do migrante interno brasileiro. Rev ABET 2009; 8(1): 125-144.

21. Soares MP. Almas e encantados: uma cosmologia sobre o mundo dos mortos na região do Baixo Amazonas [Tese]. Niterói (RJ): Universidade Federal Fluminense; 2013.

22. Coissi J. Brasil aumenta número de médicos, mas concentração continua desigual. Jornal Folha de São Paulo [Acessado 2016 ago 18]. Disponível em: http://www1.folha.uol.com.br/seminariosfolha/2015/05/1628533-brasil-aumenta-numero-demedicos-mas-concentracao-continua.shtml

23. Confalonieri UE. Saúde na Amazônia: um modelo conceitual para a análise de paisagens e doenças. Estud Av 2005; 19(53):221-236.

24. Rosa C, Camara SC, Béria, JM. Representação e intenção de uso na fitoterapia na Atenção Básica. Ciênc Saúde Coletiva 2011; 16 (1):311-318.

25. Santos RL, Guimarães GP, Nobre MSC, Portela, AS. Análise sobre a fitoterapia como prática integrativa no Sistema Único de Saúde. Rev Bras Pl Med 2011; 13(4):486-491.

26. Brasil, Ministério da Saúde, Secretaria de Gestão do Trabalho e da Educação na Saúde, Departamento de Gestão da Educação em Saúde. Política Nacional de Educação Permanente em Saúde. Portaria GM/MS nº 1.996, de 20 de agosto de 2007. Série B. Textos Básicos de Saúde v. 9. Brasília: Ministério da Saúde, 2009.

27. Brasil. Ministério da Saúde. Portaria Interministerial no 2960, de 9 de dezembro de 2008. Aprova o Programa Nacional de Plantas Medicinais e Fitoterápicos e dá outras providências. Diário Oficial da União 2008, 10 dez.

28. Oliveira DR. Levantamento Etnobotânico das Plantas Medicinais Utilizadas pela Comunidade de Oriximiná (Pará) com enfoque etnofarmacológico para o Gênero Lippia [Dissertação]. Rio de Janeiro (RJ): Universidade Federal do Rio de Janeiro; 2004.

29. Oliveira DR. Bioprospecção de espécies vegetais do conhecimento tradicional associado em comunidades quilombolas de Oriximiná-PA [tese]. Rio de Janeiro (RJ): Universidade Federal do Rio de Janeiro; 2009.

30. Agência Nacional de Vigilância Sanitária - Anvisa. Instrução Normativa $n^{\circ} 2$, de 13 de maio de 2014. Publica a "Lista de medicamentos fitoterápicos de registro simplificado" e a "Lista de produtos tradicionais fitoterápicos de registro simplificado”. Diário Oficial União. 14 mai 2014;Seção 1:58.

31. EMA - European Medicines Agency. List of the European Union Herbal Monograph [Acessado 2017 nov 26] Disponível em: http://www.ema.europa.eu/ema/index.jsp?curl=pages\%2Fmedicines\%2Flanding\%2Fherbal_search.js p\&mid=\&searchkwByEnter=false \&alreadyLoaded=true\&isNewQuery=true \&keyword=Ocimum\&ke ywordSearch=Submit\&searchType=Botanical+name+of+plant\&taxonomyPath=\&treeNumber=

32. BRASIL. RDC $\mathrm{n}^{\circ}$ 26, de 13 de Maio de 2014. Dispõe sobre o registro de medicamentos fitoterápicos e a notificação de produtos tradicionais fitoterápicos. Diário Oficial da União, Poder Executivo, Brasília, DF, 14 de Maio de 2014.

33. Oliveira DR. Oliveira ACD, Marques, LC. O estado regulatório dos fitoterápicos no Brasil: um paralelo entre a legislação e o Mercado farmacêutico (1995-2015). Vigilância Sanitária em Debate 2016; 4,(4): 139-148.

34. Menegald LR, Pereira HDS, Ferreira ADS. Os bichos e os homens: interações socioculturais com a fauna silvestre em uma comunidade amazônica. Rev Estud Amazônicos 2012; 12(1): 61-82.

35. Clarke JHR, Rates SMK, Bridi R. Um alerta sobre o uso de produtos de origem vegetal na gravidez. Infarma-Ciências Farmacêuticas 2013; 19(1/2): 41-48. 
36. Agência de Vigilância Sanitária (Anvisa). [Acessado 2016 set 29] Disponível em: http://www.anvisa.gov.br/hotsite/notivisa/index.htm

37. Fontanella F, Speck FP, Piovezan AP e Kulkamp IC. Conhecimento, acesso e aceitação das práticas integrativas e complementares em saúde por uma comunidade usuária do Sistema Único de Saúde na cidade de Tubarão-SC. Arq Catarinenses de Medicina 2007; 36(2): 69-74.

38. Ferreira LLC, Mattos, JLC, Oliveira, DR, Behrens, M. Incentivo governamental para Arranjos Produtivos Locais de Plantas Medicinais e Fitoterápicos no âmbito do SUS. Rev Fitos 2017; (Supl, 1-126): 54-61. 